\title{
Steroid hormone receptor phosphorylation: is there a physiological role?
}

\author{
G.G.J.M. Kuiper * and A.O. Brinkmann \\ Department of Endocrinology and Reproduction, Faculty of Medicine and Health Sciences, Erasmus University Rotterdam, PO Box 1738 , \\ 3000 DR Rotterdam, Netherlands
}

(Received 26 November 1993)

Key words: Hormone receptor; Steroid hormone; Transcription regulation; Protein phosphorylation; Protein kinase

\section{Summary}

All members of the steroid hormone receptor family are phosphoproteins. Additional phosphorylation occurs in the presence of hormone. This hormone-induced phosphorylation, which is 2- to 7-fold more than the basal phosphorylation, is a rapid process. All steroid receptors are phosphorylated at more than one single site. Most phosphorylation sites are located in the N-terminal domain, and phosphorylation occurs mainly on serine residues. Phosphorylation on threonine residues occurs in only a few cases. Phosphorylation on tyrosine residues has been found only for the estrogen receptor. Six different protein kinases are possibly involved in steroid receptor phosphorylation (estrogen receptor kinase; protein kinase A; protein kinase C; casein kinase II; DNA-dependent kinase; Ser-Pro kinases). Steroid receptor phosphorylation has been directly implicated in: activation of hormone binding, nuclear import of steroid receptors, modulation of binding to hormone response elements, and consequently in transcription activation.

\section{Introduction}

All members of the steroid hormone receptor family are phosphoproteins. These include the receptors for glucocorticoids, mineralocorticoids, progestins, estrogens, androgens and 1,25-dihydroxyvitamin $D_{3}$. In recent years, several detailed reviews on steroid receptor phosphorylation have appeared (Orti et al., 1992; Moudgil, 1990; Takimoto and Horwitz, 1993), and there are also several general reviews on phosphorylation of transcription factors (Meek, 1992; Hunter, 1992). The present short review focuses on recent developments and possible physiological roles of steroid receptor phosphorylation.

\section{Basal and hormone-dependent phosphorylation}

When target cells in culture or tissue slices are incubated for short or longer periods with ${ }^{32} \mathrm{P}$-orthophosphate, steroid receptors can be shown to be phosphorylated after immunopurification and SDS-polyacrylamide electrophoresis (SDS-PAGE) (Orti et al.,

* Corresponding author. Tel.: 31-10-4087966; Fax: 31-10-4366832.
1992, and references therein). This phosphorylation occurs in the absence of ligand. Another proof for hormone independent phosphorylation of steroid receptors is the fact that at least progesterone (PR), 1,25-dihydroxyvitamin $\mathrm{D}_{3}$ (VDR) and androgen (AR) receptors migrate on SDS-PAGE as multiple forms, and the number of these receptor forms can be manipulated by phosphatase treatment in cell-free systems (Sheridan et al., 1989; Jurutka et al., 1993a; Kuiper et al., 1991). On incubation of cells or tissues in culture with the respective ligands, steroid receptors become hyperphosphorylated. This extra phosphorylation is a rapid process $(<30 \mathrm{~min})$ and is associated with receptor transformation (Orti et al., 1992; Sheridan et al., 1989; Van I aar et al., 1991). This is often used as an argument to stress the importance of steroid receptor phosphorylation. The stimulation reported ranged from 2- to 7-fold (Kuiper et al., 1993; Sheridan et al., 1988; Beck et al., 1992; Mendel et al., 1987; Denton et al., 1992; Washburn et al., 1991; Brown and DeLuca, 1990). Differences reported for one type of receptor in comparable systems, might be due to variations in experimental conditions. A question which is not always addressed is whether labelling in vivo with ${ }^{32} \mathrm{P}$-orthophosphate has reached a steady state, an important 
requirement for comparison of phosphorylation levels. Results obtained with short labelling times $(<2 \mathrm{~h})$ can be useful, but tend to give prominence to rapidly turning over sites. On the other hand, most hormone sensitive phosphoproteins can be expected to equilibrate quickly with the ${ }^{32} \mathrm{Pi}$ in the ATP pool, because their phosphorylation site(s) are constantly subject to the actions of kinases and phosphatases. Thus, they will contain small amounts of ${ }^{32} \mathrm{P}$ in the unliganded state, and the phosphorylation level increases rapidly during stimulation (Garrison, 1993). Nevertheless, it usually takes $4-6 \mathrm{~h}$ before cells in culture have equilibrated the ATP-pool with the radioactive orthophosphate. Whether the increase in steroid receptor phosphorylation upon hormone treatment is the consequence of a conformational change, making the receptor a better substrate for a kinase(s) or a less good substrate for a phosphatase(s), is an open question. Direct effects of steroid hormones on the activity of kinases and/or phosphatases have not been described, but cannot be excluded.

\section{Localization of phosphorylation sites}

With regard to the localization of steroid receptor phosphorylation sites, it is now clear that progesterone, glucocorticoid, and androgen receptors are mainly phosphorylated in the amino-terminal domain (Hoeck and Groner, 1990; Sheridan et al., 1988; Denner et al., 1990; Sullivan et al., 1988; Kuiper et al., 1993,). For the chicken PR a phosphorylation site in the hinge region between the DNA and ligand binding domain has also been reported (Denner et al., 1990). The 1,25 dihydroxyvitamin $D_{3}$ receptor (VDR) is phosphorylated in a central fragment spanning the hinge region and a large part of the ligand binding domain, but also phosphorylation of a site in the DNA-binding domain has been reported (Brown and DeLuca, 1991; Hsieh et al., 1991). Multiple phosphorylation sites in the N-terminal domain have been found in the estrogen receptor. All these sites were serine residues (Ali et al., 1993). For the mineralocorticoid receptor, data on the sites of phosphorylation are not yet available, although it is known that hyperphosphorylation of this receptor by ligand binding can be induced (Alnemri et al., 1991).

\section{Identification of phosphorylation sites}

Phospho amino acid analysis has revealed that the glucocorticoid, progesterone, vitamin $\mathrm{D}_{3}$, and androgen receptors are phosphorylated on serine residues (Dalman et al., 1988; Sheridan et al., 1988; Brown and DeLuca, 1991; Kuiper, unpublished observations). In some cases minor phosphorylation on threonine residues has also been reported (Hoeck and Groner, 1990; Bodwell et al., 1991). It should be realized that phospho-esters are unstable under the conditions of acid hydrolysis of proteins, and that consequently dephosphorylation of the phospho amino acids and release of free $\left[{ }^{32} \mathrm{Pi}\right]$ occurs. Phospho-tyrosine is especially unstable, and false negative results can therefore be obtained (Duclos et al., 1991).

Results on phospho-amino acid analysis of estrogen receptors are confusing. Auricchio and co-workers have shown that calf, rat and human estrogen receptors are phosphorylated on tyrosine residues (Auricchio, 1989; Migliaccio et al., 1986). In contrast, other investigators have reported that mouse and calf uterus estrogen receptors are phosphorylated exclusively on serine residues (Washburn et al., 1991; Denton et al., 1992). The reason for these discrepancies is at present unknown.

Identification of phosphorylated residues in steroid receptors has been shown to be a major task. Large amounts of purified receptors are needed, which, are difficult to obtain, because of the very low expression level of receptor proteins in target cells. After tryptic phospho-peptide mapping and microsequencing, seven phosphorylated residues in the mouse glucocorticoid receptor were identified (Bodwell et al., 1991). All sites but one were localized in the amino-terminal transactivation domain. Data on the influence of glucocorticoids on the phosphorylation state of these sites are not yet available.

Four phosphorylated sites in the chicken progesterone receptor have been identified (Denner et al., 1990; Poletti and Weigel, 1993). There are four Ser-Pro motifs in the chicken progesterone receptor, which are all phosphorylated. Two sites are substantially phosphorylated in the absence of hormone, with a small increase in the presence of hormone, while phosphorylation of two other sites are hormone-inducible. The hormone-inducible phosphorylation sites (Ser-367 and Ser-530) are flanking the DNA-binding domain. This could explain the differential sensitivity towards hormone, since it is generally believed that the DNA-binding domain is exposed upon hormone binding, making sites available for phosphorylation.

Upon expression of chicken progesterone receptor in the yeast Saccharomyces cerevisiae, it was also shown that in this system the receptor is correctly phosphorylated on all four Ser-Pro motives (Poletti et al., 1993). The effects of hormone on the degree of phosphorylation of the four sites were consistent with those obtained in the authentic chicken PR, showing that the yeast system is appropriate for the study of the role of phosphorylation in PR function.

\section{Protein kinases and steroid receptor phosphorylation}

Progesterone receptors can be phosphorylated in vitro by several kinases. Protein kinase A phosphory- 
lates purified $\mathrm{A}$ and $\mathrm{B}$ forms of the chicken oviduct progesterone receptor on serine residues (Nakao et al., 1992). Multiple phosphopeptides were resolved by two-dimensional tryptic peptide mapping. However, no direct comparison was made with in vivo phosphorylated receptor. Chicken progesterone receptor is also phosphorylated by a DNA-dependent protein kinase during in vitro PR-dependent transcription assays using HeLa cell nuclear extracts. This phosphorylation is strictly dependent on the presence of double-stranded DNA (Weigel et al., 1992). These experiments suggest that, apart from the hormone-independent and hormone-dependent phosphorylations, an additional series of phosphorylations may be required for activation of the progesterone receptor. Further analysis of receptors isolated from chicken oviduct nuclear extracts will be necessary to determine if this phosphorylation also occurs in vivo. The DNA-dependent kinase that is involved also phosphorylates the transcription factor $\mathrm{Sp1}$, and is a heterodimer composed of a calalylic subunit and a DNA binding subunit. The latter directs the kinase to DNA and can be regarded as a "targetting subunit", making efficient phosphorylation of DNA-bound substrates possible (Hubbard and Cohen, 1993).

Cell-free phosphorylation of calf uterine estrogen receptors has been found to occur on tyrosine residues (Auricchio, 1989). A nuclear phosphatase from calf uterus inactivated hormone binding, and this dephosphorylated receptor form could be converted to an estradiol binding form again by a cytosolic calcium calmodulin-dependent tyrosine kinase (Auricchio, 1989, and references therein). This kinase has now been purified to homogeneity (Castoria et al., 1993). The uterus tyrosine kinase is a $67 \mathrm{kDa}$ protein on SDSPAGE and binds calmodulin in a $\mathrm{Ca}^{2+}$ dependent manner. In vitro the kinase phosphorylates the estradiol receptor on tyrosine, and thereby activates receptor hormone binding. By site-directed mutagenesis of tyrosine residues in the in vitro translated human estrogen receptor, tyrosine 537 was identified as the phosphorylated residue. This mulant (Tyr $\rightarrow$ Phe) is neither phosphorylated nor activated with regard to ligand binding by the kinase. Remarkably, the kinase is dependent for its activity on the presence of purified calf uterus estradiol receptor. It is an open question whether the kinase is activated by estradiol, the estradiol receptor complex or by an unknown compound in the purified receptor preparation. Furthermore, it has not yet been shown that the estradiol receptor in calf uterus in vivo is also phosphorylated on tyrosine residue 537 , and that this phosphorylation is essential for hormone-binding activation.

The vitamin $\mathrm{D}$ receptor is phosphorylated in vitro by casein kinase $\mathrm{I}$, protein kinase A (PKA) and protein kinase C (PKC) (Hsieh et al., 1991; Jurutka et al., 1993b; Jones et al., 1991). In a detailed series of experiments, it was shown that the VDR is phosphorylated in vitro as well as in vivo on serine residue 51 by PKC (Hsieh et al., 1991; Hsieh et al., 1993). Phosphopeptide mapping along with identification of the PKA and casein kinase II phosphorylation sites, utilizing site specific mutagenesis, will be required to unequivocally demonstrate the involvement of PKA and casein kinase II in the phosphorylation of VDR in vivo.

It can be concluded that six different kinases (estrogen receptor kinase, PK $\Lambda$, PKC, Casein Kinase II, DNA-dependent kinase., Ser-Pro directed kinases) have so far been reported to phosphorylate steroid receptors, and that all receptors are phosphorylated at more than one single site. Furthermore, most of the sites reported have been shown to be only partly phosphorylated. It follows that within a cell several populations of receptors exists, each with different numbers or patterns of phosphorylated sites and possibly each with different biological activities.

\section{Receptor phosphorylation and receptor function}

There is increasing evidence that gene expression is regulated by phosphorylation of transcription factors (Hunter and Karin, 1992). Since steroid receptors are known as ligand-inducible transcription factors and also exist as phosphoproteins, several studies have been performed to uncover a physiological role for receptor phosphorylation in steroid hormone action. The following receptor functions or activities linked to phosphorylation have been suggested: receptor association with heat-shock proteins, activation of hormonc-binding, nuclear import, subnuclear localization, nucleo-cytoplasmic shuttling, modulation of binding to hormone response elements, receptor-dimerization, interactions with other transcription factors, and receptor half-life (e.g. receptor turnover and recycling). Despite the fact that a number of phosphorylation sites in several steroid receptors are known, mutagenesis studies have not revealed the expected clear picture of effects on biological activity.

The estrogen receptor is the only steroid receptor which is phosphorylated on a tyrosine residuc in the hormone-binding domain (Auricchio, 1989). Extensive in vitro studies have demonstrated that this phosphorylation is dependent on the estradiol-receptor complex and is activated by a $\mathrm{Ca}^{2+}$ calmodulin-dependent kinase. Phosphorylation in vitro of the dephosphorylated estrogen receptor is essential for activation of hormone-binding (Castoria et al., 1993).

In sharp contrast to these studies, there are several observations on estrogen receptor phosphorylation only on serine residues. Denton et al. (1992) have reported that the ER from the MCF-7 breast carcinoma cell line, as well as the ER from calf uterine slices, was 
phosphorylated in the presence of hormone only on serine residues, and that treatment with phosphatase caused loss of affinity of the ER for specific DNA sequences. Activation of transcription by the rat estrogen receptor can be stimulated 8-to 10 -fold by $8-\mathrm{Br}-$ cAMP, cholera toxin and insulin-like growth factor $\mathrm{I}$. These agents also elicit a 3- to 5-fold increase in overall phosphorylation of the receptor (Aronica and Katzenellenbogen, 1993). However, the fact that an anti-estrogen evokes a similar increase in ER phosphorylation, without a similar increase in ER activation of transcription, indicates that an increase in overall phosphorylation does not necessarily result in increased transcriptional activity. Transactivation of the ER, therefore, might depend upon phosphorylation of a specific site or sites, the modulation of which might not be detected in overall phosphorylation. In another study involving transfection of COS cells with ER, multiple phosphorylation sites in the $\mathrm{N}$-terminal domain were found, all on serine residues (Ali et al., 1993). Mutation of one serine residue (position 118 in the human estrogen receptor) caused a significant reduction in transcription activation by the receptor, but did not affect the DNA-binding properties or nuclear import of the receptor. The serine residue at position 118 co-localizes with the transcription activation function 1 (AF-1) in the $\mathrm{N}$-terminal domain (Ali et al., 1993).

Kinetic data on in vitro transcription by the hPR has revealed a positive correlation between progesteronedependent phosphorylation of the PR and the progesterone induced enhancement of RNA synthesis from a PRE-driven promoter (Bagchi et al., 1992). Although in the chicken progesterone receptor four different phosphorylation sites have been identified, no data on functional significance are available yet. Hyperphosphorylation of the progesterone receptor is not correlated with increased transcriptional activity, because the antagonist RU486 provokes hyperphosphorylation in the rabbit progesterone receptor, whereas a constitutively active progesterone receptor mutant is not hyperphosphorylated (Chaucherau et al., 1991). Careful comparison by two-dimensional tryptic phosphopeptide mapping could resolve the differences in phosphorylation of the PR after treatment with RU486 or progesterone.

In the mouse glucocorticoid receptor, seven phosphorylation sites have been identified (Bodwell et al., 1991). Single mutations at all seven sites, or combinations of substitutions, elicited levels of hormone-induced reporter gene expression that are comparable to wild type receptors (Mason and Housley, 1993). From these studics, it can be concluded that phosphorylation of the GR at the seven identified sites is not a major determinant in GR mediated transcription activation. Other studies with the GR indicate that receptor phos- phorylation could affect intracellular trafficking. Nuclear retention is inhibited by several phosphatase inhibitors, but not nuclear uptake. Dephosphorylation of the GR might promote cytoplasmic trapping of the GR. Hormone-dependent phosphorylation of the GR may in fact serve as a mechanism for regulating the intracellular distribution, via changes in nucleo-cytoplasmic shuttling (Hsu et al., 1992; Orti et al., 1993) Phosphorylation of various components of the GR signal transduction pathway, and not necessarily the receptor itself, may influence its transcriptional enhancement activity (DeFranco et al., 1991; Moyer et al., 1993).

The human vitamin $\mathrm{D}$ receptor is selectively phosphorylated by protein kinase $\mathrm{C}$ on serine at position 51 . This serine residue lies in a position between the two zinc fingers that constitute the DNA binding domain. Mutation of the serine residue at position 51 to a negatively charged aspartic acid residue markedly reduced the binding of the VDR to a vitamin D responsive element and consequently the transcriptional activity (Hsieh et al., 1993). Alteration of serine 51 to alanine in the hVDR eliminated phosphorylation but preserved completely the specific DNA binding activity and transactivation capacity. It has been suggested that phosphorylation at serine 51 could silence the hVDR via a negative regulation of DNA binding when target cells are subject to PK-C activation events (Hsieh et al., 1993).

\section{Conclusions}

For all steroid receptors, overwhelming evidence is reported for hyperphosphorylation of the receptor after ligand binding. In some cases, this can result in increased transcriptional activity. Phosphorylation can increase the negative charge and acidity of a region of a protein, thereby modifying interactions with other proteins or with DNA. Hypo- and hyperphosphorylation at the same time in different regions of steroid receptor molecules might provide a mechanism for differential transcription rcgulation of certain genes, in addition to host cell and promoter context of the genes to be transcribed or repressed. The possible role of altered phosphorylation states of steroid receptors may be illustrated by studies with SV 40 large $T$ antigen and c-fos in which differential phosphorylation produced different cellular actions (Prives, 1990; Ofir et al., 1990). The fact that there are so many phosphorylation sites affected by different kinases/phosphatases and signal transduction pathways, might indicate that phosphorylation is involved in the regulation of multiple receptor functions. In this respect, a further identification of kinases and phosphatases involved in steroid receptor phosphorylation/dephosphorylation, as well as their regulation, are necessary for a better under- 
standing of the role of receptor phosphorylation in steroid hormone action. Additional mutagenesis studies of phosphorylation sites in vitro and in vivo (homologous recombination experiments), and structural analysis studies at the three-dimensional level (X-ray analysis and NMR studies), might help further to elucidate and understand the consequences of the introduction of a negative phosphate group for the structure and activity of these ligand-induced Iranscription factors.

\section{Acknowledgement}

The authors wish to thank Dr. J.A. Grootegoed for reviewing the manuscript.

\section{References}

Ali, S., Metzger, D., Bornert, J.M. and Chambon, P. (1993) EMBO J. $12,1153-1160$.

Alnemri, E.S., Maksymowych, A.B., Robertson, N.M. and Litwack, G. (1991) J. Biol. Chem. 266, 18072-18081

Aronica, S.M. and Katzenellenbogen, B.S. (1993) Mol. Endocrinol. 7, 743-752.

Auricchio, F. (1989) J. Steroid Biochem. 32, 613-622.

Bagchi, M.K., Tsai, S.Y., Tsai, M.J. and O'Malley, B.W. (1992) Proc. Natl. Acad. Sci. USA 89, 2664-2668.

Beck, C.A., Weigel, N.L. and Edwards, D.P. (1992) Mol. Endocrinol. $6,607-620$.

Bodwell, J.E., Orti, E., Coull, J.M., Pappin, D.J.C., Smith, L.I. and Swift, F. (1991) J.Biol. Chem. 266, 7549-7555.

Brown, T.A. and DeLuca, H.F. (1990) J. Biol. Chem. 265, $10025-$ 10029.

Brown, T.A. and DeLuca, H.F. (1991) Arch. Biochem. Biophys. 286, 466-472.

Castoria, G., Migliaccio, A., Green, S., Di-Domenico, M., Chambon, P. and Auricchio, F. (1993) Biochemistry 32, 1740-1750.

Chauchereau, A., Loosfelt, H. and Milgrom, E. (1991) J. Biol. Chem. 266, 18280-18286.

Dalman, F.C., Sanchez, E.R., Lin, A.L.Y, Perini, F. and Pratt, W.B. (1988) J. Biol. Chem. 263, 12259-12267.

DeFranco, D.B., Qi, M., Borror, K.C., Garabedian, M.J. and Brautigan, D.L. (1991) Mol. Endocrinol. 5, 1215-1228.

Denner, L.A., Schrader, W.T., O'Malley, B.W. and Weigel, N.L. (1990) J. Biol. Chem, 265, 16548-16555.

Denton, R.R., Koszewski, N.J. and Notides, A.C. (1992) J. Biol. Chem. 267, 7263-7268.

Duclos, B., Marcandier, S. and Cozzone, A.J. (1991) Methods Fnzymol. 201, 10-21.

Garrison, J.C. (1993) in Protein Phosphorylation (Hardie, G., ed), pp. 1--28, IRL Press, Oxford.

Hoeck, W. and Groner, B. (1990) J. Biol. Chem. 265, 5403-5408.

Hsieh, J.C., Jurutka, P.W., Galligan, M.A., Terpening, C.M., Haussler, C.A., Samuels, D.S., Shimizu, Y., Shimizu, N. and Haussler, M.R. (1991) Proc. Natl. Acad. Sci. U.S.A. 88, 9315-9319.
Hsich, J.C., Jurutka, P.W., Nakajima, S., Galligan, M.A., Haussler, C.A., Shimizu, Y., Shimizu, N., Whitfield, G.K. and Haussler, M.R. (1993) J. Biol. Chem. 268, 15118-15126.

Hsu, S.C., Qi, M. and DeFranco, D.B. (1992) EMBO J. 11, 34573468.

Hubbard,M.J. and Cohen, P. (1993) Trends Biochem. Sci. 18, 172177.

Jones, B.B., Jurutka, P.W., Haussler, C.A., Haussler, M.R. and Whitfield, G.K. (1991) Mol. Endocrinol. 5, 1137-1146.

Hunter, T. and Karin, M. (1992) Cell 70, 375-387.

Jurutka, P.W., Terpening, C.M. and Haussler, M.R. (1993a) Biochemistry $32,8184-8192$.

Jurutka, P.W., Hsieh, J.C., MacDonald, P.N., Terpening, C.M., Haussler, C.A., Haussler, M.R. and Whitfield, G.K. (1993b) J. Biol. Chem. 268, 6791-6799.

Kuiper, G.G.J.M., De Ruiter, P.E., Grootegoed, J.A. and Brinkmann, A.O. (1991) Mol. Cell. Endocrinol. 80, 65-73.

Kuiper, G.G.J.M., De Ruiter, P.E., Trapman, J., Boersma, W.J., Grootegoed, J.A. and Brinkmann, A.O. (1993) Biochem. J. 291, 95-101.

Mason, S.A. and Housley, P.R. (1993) J. Biol. Chem. 268, 2150121504.

Meek, D.W. and Street, A.J. (1992) Biochem. J. 287, 1-15.

Mendel, D.B., Bodwell, J.E. and Munck, A. (1987) J. Biol. Chem. 263, 6695-6702.

Migliaccio, A., Rotondi, A. and Auricchio, F. (1986) EMBO J. 5, 2867-2872.

Moudgil, V.K. (1990) Biochim. Biophys. Acta 1055, 243-258.

Moyer, M.L., Borror, K.C., Bona, B.J., DeFranco, D.B. and Nordcen, S.K. (1993) J. Biol. Chem. 268, 22933-22940.

Nakao, M., Mizutani, T., Bhakta, A., Ribarac-Stepic, N. and Moudgil, V.K. (1992) Arch. Biochem. Biophys. 298, 340-348.

Ofir, R., Dwarki V.J., Rashid, D. and Verma, I. (1990) Nature 348, $80-82$.

Orti, E., Bodwell, J.E. and Munck, A. (1992) Endocrine Reviews 13, $105-128$.

Orti, E., Hu, L.M. and Munck, A. (1993) J. Biol. Chem. 268, $7779-7784$.

Poletti, A. and Weigel, N.L. (1993) Mol. Endocrinol. 7, 241-247.

Poletti, A., Conneely, O.M., McDonnell, D.P.. Schrader, W.T., O'Malley, B.W. and Weigel, N.L. (1993) Biochemistry 32, 9563 9569.

Prives, C. (1990) Cell 61, 735-738.

Sheridan, P.L., Krett, N.L., Gordon, J.A. and Horwitz, K.B. (1988) Mol. Endocrinol. 2, 1329-1342.

Sheridan, P.L., Francis, M.D. and Horwitz, K.B. (1989) J. Biol. Chem. 264, 7054-7058.

Sullivan, W.P., Madden, B.J., McCormick, D.J. and Toft, D.O. (1988) J. Biol. Chem. 263, 14717-14723.

Takimoto, G.S. and Horwitz, K.B. (1993) Trends Endocrinol. Metabolism 4, 1-7.

Van Laar, J.H., Berrevoets, C.A., Trapman, J., Zegers, N.D. and Brinkmann, A.O. (1991) J. Biol. Chem. 266, 3734-3738.

Washburn, T., Hocutt, A., Brautigan, D.L. and Korach, K.S. (1991) Mol. Endocrinol. 5, 235-242.

Weigel, N.L., Carter, T.H., Schrader, W.T, and O'Malley, B.W. (1992) Mol. Endocrinol. 6, 8-14. 